\title{
NEW ANALYTICAL METHOD TO DESIGN FILTERS BY COMBINING THE PERIODIC AND RESONANT FILTERING EFFECTS OF EBG CELLS
}

\author{
A. Ibáñez and C. del-Río \\ Antenna Group, Universidad Pública de Navarra, \\ Campus Arrosadía s/n, 31006 Pamplona, Spain. \\ Phone: +34 948169326 \\ E_mail: carlos@unavarra.es
}

In this paper a new analytical method to design stop-band filters combining the Bragg and resonant effects is presented. A rigorous consideration of the EBG structures filtering effect at the microwave frequency range will be performed. We will show that in that frequency range, the periodic structure filtering effect (or Bragg effect) and the self-resonance of the different cells involved are tuned approximately at the same frequencies, mixing their influences in the final filter response. Both effects are defined by different geometrical laws, and filters with very interesting properties such as high rejections, sharpness and reduced insertion losses, can be obtained when tuning the EBG effect and the resonant effect at the same frequency.

\section{Introduction}

It is really amazing the amount of publications that monthly present new possibilities to design filters in microstrip, strip-line, or any other planar technology using EBG/PBG (Electromagnetic Band Gap/Photonic Band Gap) or DGS (Defected Ground Structures) structures. Usually holes or defects are performed on the ground plane $[1,2,3]$, or some kind of special stubs or parasitic elements are added to the same transmission line [4]. However none of these papers present a rigorous analytical method to fully design such filters. In most cases, the EBG response of the combination of unit cells is mistaken with the resonant response of each unit cells, due the coexistence of both effects in the same frecuencial range because of the similar physical dimensions involved in each effect.

In this paper, we will show that at microwave frequency range, we will encounter two different kind of responses when designing stopband filters, the one caused by the EBG effect (ruled by the distance between cells) and the other one caused by the self-resonances (ruled by the physical dimensions of each cell).

We want to emphasize the new idea of analyze and synthesize both effects separately to combine them in order to obtain stop band filters with high rejections and sharpness with reduced insertion losses. Probably this conclusion of tuning at the same frequency both effects, have been obtained many times from some GA algorithms or optimization codes. In this paper, we are characterizing the consequences of this combination and providing a concrete analytical design method to obtain quasi-optimum filter designs that full-fill all the bandwidth requirements.

\section{Analysis of a simple example}

To show the above exposed idea, and in order to achieve a better understanding of the physical phenomena involved in EBG and resonant effects, various microwave circuits have been designed and simulated, using shunt stubs, classical and well known structures in the field of stopband filter design, figure 1.

By using stubs, we want to maximize the clearness of our analysis, having not to deal with complex unit cells with a large amount of parameters to characterize them.

The proposed circuit has been designed using eight shunt stubs, in order to obtain a frequency response with finite attenuation poles in the resonance frequency of the stubs. The geometrical parameters of the circuit are: $\mathrm{L}=2 \mathrm{~mm}, \mathrm{~W}=0.5 \mathrm{~mm}$ and $\mathrm{D}=8$ $\mathrm{mm}$. The width of the transmission line has been chosen to be $1.16 \mathrm{~mm}$ in order to 
obtain a good impedance matching in the pass band. The chosen technology is microstrip with a dielectric constant $\varepsilon=10.2$ and a thickness of $1.27 \mathrm{~mm}$.

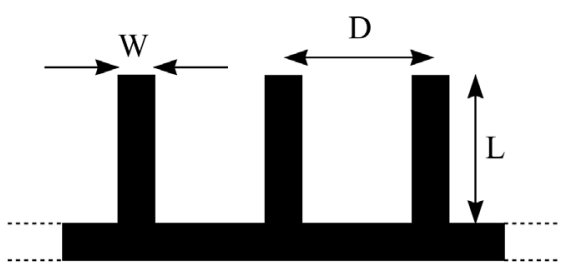

Figure 1.- Shunt stub filter technology.

In the simulated response, figure 2 , it can be seen very clearly the independence of both EBG and resonant effects, having the EBG response at $6.5 \mathrm{GHz}$ (smooth and rounded) and the resonant response at $11.5 \mathrm{GHz}$.

The EBG response is caused by the periodic disposition of the unit cells (in our case, stubs). On the other hand, the resonant frequency is ruled by geometrical parameters of the unit cells (in our case, the stub length).

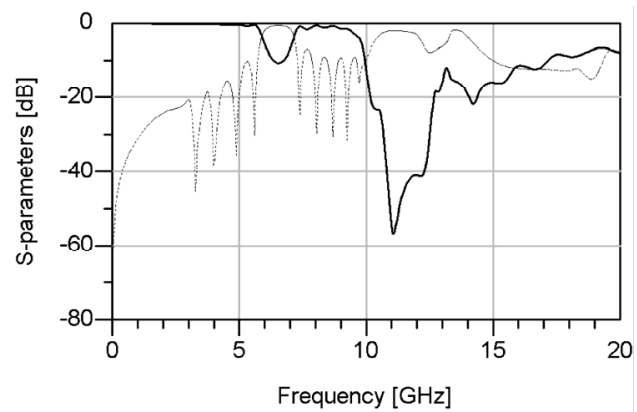

Figure 2.- $\mathrm{S}_{21}$ (continuous) and $\mathrm{S}_{11}$ (dashed) simulated parameters of one of the stubs circuits. It can be seen the EBG effect at 6.5 $\mathrm{GHz}$ and the resonance effect at $11 \mathrm{GHz}$.

In order to prove that the two effects are ruled by different physical parameters, and that can be easily tuned separately, two sets of circuits have been simulated. In each sets of circuits, only one geometrical parameter is modified, in order to isolate its influence in the circuit response.

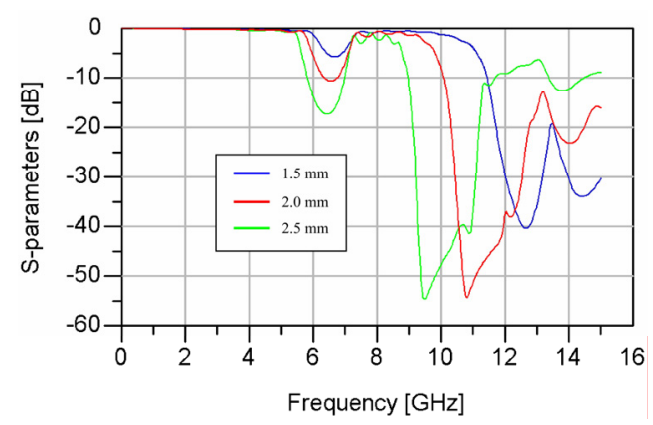

Figure 3.- $\mathrm{S}_{21}$ simulated parameter of three different shunt stub filters changing the stub lengths.

Figure 3 shows the result of varying the length of the stubs (parameter L) from 1.5 $\mathrm{mm}$ to $2.5 \mathrm{~mm}$. It can be seen that the resonant response of the stubs lowers its frequency as we increase the length of the stubs as it could be expected. At the same time, the EBG filtering response maintains its frequency.

Now, if we maintain the stub lengths and vary the separation between them (parameter D) from $10 \mathrm{~mm}$ to $12 \mathrm{~mm}$, we will be modifying the period of the whole structure, and the frequency at which it satisfies the Bragg condition and produces the EBG rejection. Figure 4 shows the simulated results, and it can be observed that the resonances remain at $12 \mathrm{GHz}$ as we expected, while the EBG rejection changes its frequency from $4.5 \mathrm{GHz}$ to $5.5 \mathrm{GHz}$. The periodicity of the EBG response can also be observed, showing its second harmonic between $8 \mathrm{GHz}$ and $10 \mathrm{GHz}$.

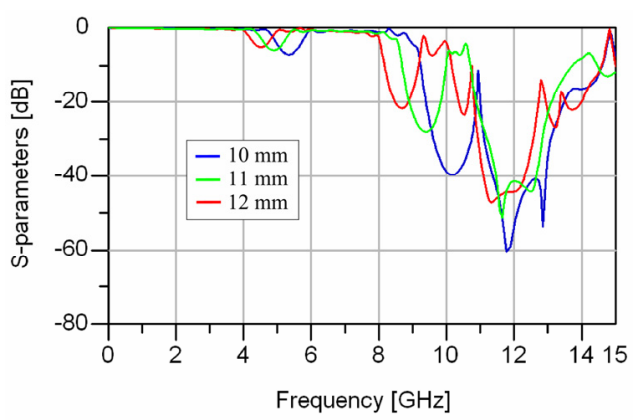

Figure 4.- $\mathrm{S}_{21}$ simulated parameter of three different shunt stub filters changing the stub separation.

It has been demonstrated that the distance between the cells is that truly rules the 
rejecting frequency of the EBG effect, and that the geometrical parameters of the unit cells rule the resonant response of the cells, and only affect the EBG response in its rejection depth.

\section{Analytical Design Process}

The new proposed design process it is based on the characterization of the unit cells individual resonant response. That response will be characterized with its rejecting frequency and its rejecting $3 \mathrm{~dB}$ bandwidth. With those two parameters, the quality factor of the resonator can be easily calculated as the inverse of the fractional bandwidth of the resonance response.

In figure 5, it can be seen the relation between the quality factor of the resulting filter of combining the EBG and resonant response and the quality factor of the individual resonant cells.

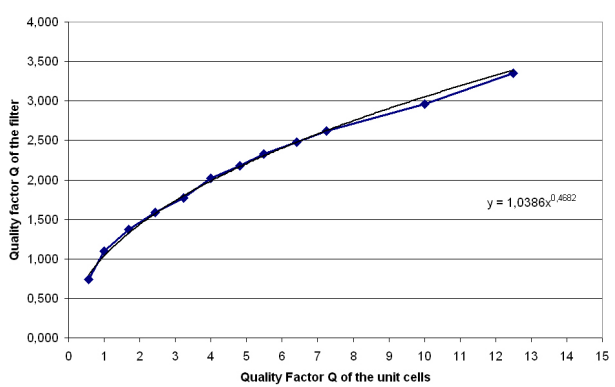

Figure 5.- Relation between the rejection band quality factor of the whole filter, and the one of each unit cell.

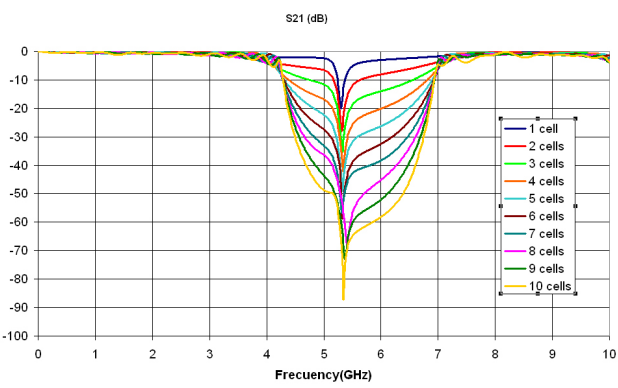

Figure 6.- $\mathrm{S}_{21}$ simulated for different filters combining EBG and resonant effects at $5.5 \mathrm{GHz}$, varying the number of cells considered.

With that relation, it is easy to derive the quality factor of the unit cells needed, in order to meet certain bandwidth requirements of the final filter. The rest of the process only requires tuning the resonances of the unit cells to be the central frequency of the filter, and separating that unit cells $\lambda / 2$ at the same frequency.

The number of cells included in the filter will only affect the rejection level at the stop-band filter, that it will be increased as the number of cells increase, figure 6 .

In order to show the above exposed and that both effects can be easily combined in a single filter, an 8 to $12 \mathrm{GHz}$ stopband filter has been designed, simulated, built and measured, using the proposed design method, figure 7. Only 10 unit cells have been used in the circuit, and the stopband filter has the expected good properties like large rejection and sharpness, and reduced insertion looses.

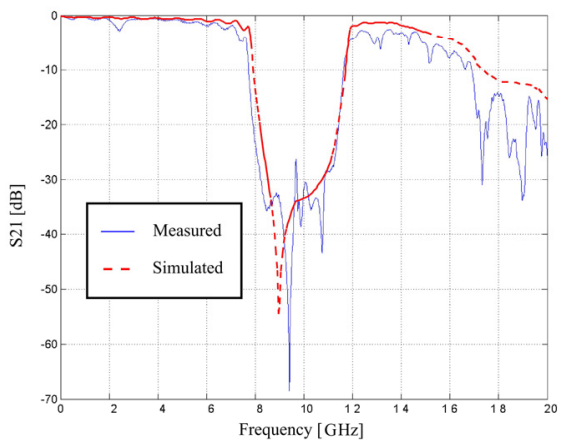

Figure 7.- $\mathrm{S}_{21}$ simulated (dashed) and measured (continuous) parameters of the 8 to $12 \mathrm{GHz}$ stop-band filter with EBG and resonant effect tuned at the same frequency.

\section{Conclusions}

In the performed simulations and measurements, the coexistence of the EBG response of a periodic structure of cells and the resonant response of each cell has been shown. When designing a filter in microwave ranges using periodic structures, we must take into account the effects of the resonances of the cells employed.

Combining both EBG and resonant responses at the same frequency provides stop band filtering responses with very good properties like sharpness and reduced insertion loss, as well as high rejections with a reduced number of periods unlike classical EBG filters.

Finally, an analytical procedure based on 
relating the quality factor of the cells and the desired one of the final filter has been presented.

\section{References}

[1] C.S Kim, J.S. Park, D. Ahn, and J.B. Lim, A novel 1-D periodic defected ground structure for planar circuits, IEEE Mwave Guided Wave Letters 10, 2000.

[2] Ieltxu A. Garde Irigoyen and Carlos del Río Bocio, A stopband filter design using a $1 \mathrm{D}$ non-periodic defected ground structure, IEEE Mwave and Optical Technology Letters Vol. 36, No. 4, 2003.

[3] Ieltxu Garde, Miguel Javier Yábar, and Carlos del Río, Simple modelling of DGS to design 1D-PBG low-pass filters, IEEE Mwave and Optical Technology Letters Vol. 37, No. 3, 2003.

[4] Dusan Nesic, A new type of slow-wave 1D PBG microstrip band-pass filter, IEEE Mwave and Optical Technology Letters Vol. 37, No. 3, 2003. 